\title{
Sexual Minority Individuals and Pregnancy Outcomes: A Commentary
}

\author{
Julie Croll ${ }^{1}$, Laura Sanapo ${ }^{2}$, and Ghada Bourjeily ${ }^{2}$ \\ ${ }^{1}$ Royal College of Surgeons in Ireland \\ ${ }^{2}$ Warren Alpert Medical School of Brown University
}

December 3, 2021

\section{Sexual Minority Individuals and Pregnancy Outcomes: A Commentary}

Julie Croll, MPH${ }^{\mathrm{a}, \mathrm{b}}$; Laura Sanapo, MD, MSHS, RDMS ${ }^{\mathrm{a}, \mathrm{c}}$; Ghada Bourjeily, MD, FCCP ${ }^{\mathrm{a}, \mathrm{c}}$

a'Women's Medicine Collaborative at Lifespan, The Miriam Hospital, 146 West River Street, Providence, RI 02904, USA

${ }^{\mathrm{b}}$ RCSI University of Medicine and Health Sciences, School of Medicine, 123 St Stephen's Green, Dublin 2, Ireland

${ }^{c}$ Warren Alpert Medical School of Brown University, Department of Medicine, 222 Richmond Street, Providence, RI, USA

\section{Corresponding Author}

Julie Croll, MPH

Women's Medicine Collaborative at Lifespan

146 West River Street

Providence, RI 02904, USA

E-mail: juliecroll@rcsi.com

\section{Word Count}

Title: 8 words

Main text: 1411 words

Tables: 1

References: 16

Brief title: Sexual Minority Individuals and Pregnancy Outcomes

\section{Introduction}

Sexual minority individuals who are pregnant or want to become pregnant face worse pregnancy outcomes than heterosexual patients. ${ }^{1,2}$ The American College of Obstetricians and Gynecologists (ACOG) endorses quality care to all people regardless of sexual orientation or gender identity. ${ }^{3,4}$ This includes providing gender affirming care throughout a patient's pregnancy, acknowledging risk factors for diverse sexual minority populations that affect reproductive outcomes, and utilizing appropriate evidence-based clinical recommendations 
to tailor care. ${ }^{3,4}$ Despite that, these patients still encounter many barriers to health care and have limited access to obstetric care and fertility clinics. ${ }^{3,5}$ As clinicians and scientists, it is crucial that we continue to promote equitable care to pregnant individuals of any background.

The aim of this commentary is to describe pregnancy outcomes of sexual minority individuals, identify research gaps, describe clinical perspectives, and propose future directions from a research, clinical care, and educational perspective.

\section{Pregnancy outcomes for lesbian and bisexual women}

Sex and gender minority individuals comprise a vast range of sexual preferences that contribute to their specific reproductive needs. ${ }^{6}$ Worse outcomes have been reported in sex and gender minority individuals in both their ability to achieve a successful pregnancy, and in perinatal outcomes. Pregnancy success rates are overall poorer as compared to heterosexual women. For lesbian and bisexual women, the pregnancy success rate overall is greatly reduced (9-fold lower in lesbian and 2-fold lower in bisexual patients) as compared to heterosexual women, despite parental wishes to become pregnant. ${ }^{2,7}$ Additionally, sexual minority women are over 12 times more likely to use fertility treatments, with up to $80 \%$ of same-sex couples using anonymous sperm donors. ${ }^{8}$ Though there are higher success rates of reproductive assistance in lesbians as compared to heterosexual women, both lesbian and bisexual populations reported increased rates of preterm birth (OR 1.84, 95\% CI 1.11-3.04) and stillbirth (OR 2.85, 95\% CI 1.34-2.35). ${ }^{1}$ Pregnant lesbian and bisexual patients also exhibit higher risk for depression (OR 2.85, 95\% CI 1.47-5.52) and mental distress with onset in pregnancy (OR 3.13, 95\% 1.45-6.75), and are at a higher risk for pre-existing chronic medical conditions (OR 2.09, 95\% CI 1.11-3.93) as compared to pregnant heterosexual women, after adjusting for sociodemographic factors. ${ }^{5}$ Of note, sexual minority women have equal to or higher rates of unintended pregnancies as compared to heterosexual women, and significantly higher rates of sexual violence. ${ }^{9}$

\section{Risk factors for poor obstetric outcomes among lesbian and bisexual women}

Risk factors for poor pregnancy outcomes among lesbian and bisexual women remain poorly understood. It has been hypothesized that discrepancies in these outcomes are related to disenfranchised sexual minority status, low socioeconomic status, limited access to health care and health insurance, and limited healthcare services equipped to treat the specific needs of these populations. ${ }^{3,}{ }^{10}$ Even prior to conception, sexual minority women are reported to underutilize routine health care services, including basic services such as screening for sexually transmitted infections, or attending influenza vaccination clinics. ${ }^{5}$ All of these factors negatively affect the pregnancy outcomes of lesbian and bisexual women.

Pregnancy outcomes for transgender and non-binary parents

Much less is known about pregnancy outcomes for transgender and non-binary parents, and many other sex and gender minority populations are left out of the literature entirely. ${ }^{5}$ Studies show that transgender men face many obstacles to pregnancy. Barriers include hormone therapy, fertility preservation, birth trauma, gender dysphoria, difficulties in post-partum care, as well as underutilization of contraception, lack of abortion access, and care from healthcare professionals who are equipped to manage their complex hormonal and psychosocial needs prior to, and after birth. ${ }^{10,}{ }^{11}$ There is less known about non-binary individuals. ACOG has recently recognized how marginalization of transgender and gender diverse communities leads to poor health outcomes, and acknowledged the need to improve training amongst healthcare providers regarding specific reproductive needs of these diverse populations in order to reduce inequities that have traditionally limited access to inclusive healthcare. ${ }^{4}$

\section{Research gaps}

The current literature presents several limitations on this topic. The terminology used over the past ten years to define sexual minority individuals has been quite heterogeneous and inconsistent limiting the ability to perform adequate data synthesis to assess the state of the science, identify gaps or suggest adequate clinical recommendations. ${ }^{4}$ Lack of consistent terminology also affects the possibility of performing national population studies. Only in 2020, the United States Census Bureau attempted to remedy the lack of 
data on sexual minority individuals, by including responses from the lesbian, gay, bisexual, transgender, or queer community and persons in a same-sex relationship. ${ }^{12}$ Additional limitations are that previous studies have focused more on sexual behaviors and risk of pregnancy rather than health, access to care, or pregnancy outcomes in this population. ${ }^{5}$ Other factors to be considered are the paucity of prospective studies on this research topic, and difficulty in recruiting sexual minority patients due to their limited access to health care and academic centers, as well as possible stigma that these individuals experience. ${ }^{4}$ All of these limitations affect the information and knowledge available to health care providers, advocates, policymakers, and researchers. For the healthcare provider, this translates into limited training. In 2018, less than half of board-certified American obstetricians-gynecologists reported having any training with regards to care for sexual minority patients. ${ }^{10}$

\section{Clinical perspectives}

From a clinical perspective, it is important to emphasize that sexual minority individuals may approach perinatal and reproductive care with higher levels of anxiety compared to heterosexual women. ${ }^{13}$ Both sexual minority and heterosexual pregnant patients may experience fear of childbirth, which refers to fear caused by different events, such as becoming pregnant, being pregnant, or giving birth. ${ }^{13}$ However, sexual minority individuals may also experience discrimination which can ultimately lead to higher levels of perinatal stress. ${ }^{13}$ Therefore, prenatal clinics should be more inclusive, as recently advocated by $\mathrm{ACOG}^{4} \mathrm{This}_{\mathrm{c}}$ can be obtained by promoting training and education among all medical and non-medical staff, regarding aspects of a prenatal care visit of sexual minority individuals. Added to the above stressors are the logistical issues of financing reproductive care: from assisted reproductive technology procedures like in vitro fertilization to finding healthcare professionals trained in the diverse needs of this population, pregnancy for sexual minority individuals is costly. 1,7 The creation of multidisciplinary services and collaboration with policymakers to make prenatal care more affordable is crucial, similarly to what has been advocated for transgender and nonbinary pediatric patients. ${ }^{14}$ Multidisciplinary prenatal clinics in this population would include obstetricians, midwives, lactation consultants, psychologists, psychiatrics, social workers and medical subspecialists as needed, with expertise in pregnant sexual minority individuals, and would facilitate the delivery of much needed physical and mental health services.

\section{Future directions}

Based on the described gaps in clinical care, research, and educational, we propose several key points for future directions in these areas (Box 1). It is essential for researchers to educate themselves on the use of the correct terminology more consistently in order to curate future knowledge that would be of value to clinicians. Research priorities would include the examination of discrepancies in pregnancy outcomes and associated predictors and risk factors, utilize qualitative methods to examine prenatal care experiences and identify barriers to care, and assess interventions aimed at improving access to care as well as perinatal and pregnancy success outcomes. Similarly to mandates to include women and sex as a biological variable in research proposals instituted in the past couple of decades by some governmental funding bodies, sexual minority individuals should only be excluded for scientific reasons rather than convenience. In regard to clinical care, efforts should be made to create an inclusive environment, with presence of multidisciplinary clinical teams at least at larger medical centers to provide appropriate patient care before, during and after pregnancy. From an educational point of view, training on sex and gender minority individuals should be incorporated in medical school curricula, in obstetrics and gynecology graduate medical education, and into subspecialty care at all levels. Finally, from a societal standpoint, we need to rise above societal stigmas that feed into the disenfranchisement of this population and impact health in general, and mental health of sexual minority populations.

Research Require justification for exclusion of sexual minority individuals from studies Fund studies focused on perinatal

Box 1. Key points for future directions in gender inclusive research, care, and education in reproductive health of sexual minority individuals 


\section{Concluding remarks}

Equitable prenatal care for sex and gender minority individuals is not a given with regards to the pregnant patient. While physicians are bound to do no harm, disparities in reproductive healthcare remain prevalent. ${ }^{1,4,10}$ Sexual minority individuals deserve the same level of reproductive autonomy and healthcare access that is given to heterosexual patients, and we must continue to build the physical, emotional, and psychosocial structures necessary to provide comprehensive gender affirming quality care.

\section{Acknowledgements}

The authors have no acknowledgements to disclose.

\section{Disclosure of Interests}

Dr. Boujeily has received grant funding from the National Institute of Child Health and Human Development (NICHD) and the National Heart, Lung, and Blood Institute (NHLBI).

Dr. Sanapo has received grant funding from the Rhode Island Foundation.

\section{Conflicts of interest}

The authors have no conflicts of interest to disclose.

\section{Contribution to Authorship}

Concept and design: JC and LS. Writing and preparation of manuscript: JC, LS, and GB. Critical revision of the manuscript for intellectual content: JC, LS, and GB. Administrative, technical, or material support: JC, LS, and GB.

\section{Funding}

None

\section{References}

1. Everett BG, Kominiarek MA, Mollborn S, Adkins DE, Hughes TL. Sexual Orientation Disparities in Pregnancy and Infant Outcomes. Matern Child Health J. 2019 Jan;23(1):72-81.

2. Hodson K, Meads C, Bewley S. Lesbian and bisexual women's likelihood of becoming pregnant: a systematic review and meta-analysis. BJOG. 2017 Feb;124(3):393-402.

3. ACOG Committee Opinion No. 525: Health care for lesbians and bisexual women. Obstet Gynecol. 2012 May;119(5):1077-80.

4. Health Care for Transgender and Gender Diverse Individuals: ACOG Committee Opinion, Number 823. Obstet Gynecol. 2021 Mar 1;137(3):e75-e88.

5. Gonzales G, Quinones N, Attanasio L. Health and Access to Care among Reproductive-Age Women by Sexual Orientation and Pregnancy Status. Womens Health Issues. 2019 Jan-Feb;29(1):8-16.

6. Januwalla AA, Goldberg AE, Flanders CE, Yudin MH, Ross LE. Reproductive and Pregnancy Experiences of Diverse Sexual Minority Women: A Descriptive Exploratory Study. Matern Child Health J. 2019 Aug;23(8):1071-1078.

7. Carpenter E, Everett BG, Greene MZ, Haider S, Hendrick CE, Higgins JA. Pregnancy (im)possibilities: identifying factors that influence sexual minority women's pregnancy desires. Soc Work Health Care. 2020 Mar;59(3):180-198.

8. Downing JM. Pathways to pregnancy for sexual minority women in same-sex marriages. Am J Obstet Gynecol. 2019 Sep;221(3):281-282. 
9. Jones RK, Jerman J, Charlton BM. Sexual Orientation and Exposure to Violence Among U.S. Patients Undergoing Abortion. Obstet Gynecol. 2018 Sep;132(3):605-611.

10. Mehta PK, Easter SR, Potter J, Castleberry N, Schulkin J, Robinson JN. Lesbian, Gay, Bisexual, and Transgender Health: Obstetrician-Gynecologists' Training, Attitudes, Knowledge, and Practice. J Womens Health (Larchmt). 2018 Dec;27(12):1459-1465.

11. Greenfield M, Darwin Z. Trans and non-binary pregnancy, traumatic birth, and perinatal mental health: a scoping review. International Journal of Transgender Health. 2020;22(1-2):203-216.

12. U.S. Census Bureau. 2020 Census: LGBTQ+. 2021 [cited 2021 November 11]; Available from: https://www.census.gov/newsroom/press-kits/2020/2020-census-lgbtq.html

13. Malmquist A, Jonsson L, Wikström J, Nieminen K. Minority stress adds an additional layer to fear of childbirth in lesbian and bisexual women, and transgender people. Midwifery. 2019 Dec;79:102551.

14. Inwards-Breland DJ, Karrington B, Sequeira GM. Access to Care for Transgender and Nonbinary Youth: Ponder This, Innumerable Barriers Exist. JAMA Pediatr. 2021 Nov 1;175(11):1112-1114.

15. Utamsingh PD, Kenya S, Lebron CN, Carrasquillo O. Beyond sensitivity. LGBT healthcare training in US medical schools: A review of the literature. American Journal of Sexuality Education. 2017;12(2):148-169.

16. Bonvicini KA. LGBT healthcare disparities: What progress have we made? Patient Education and Counseling. 2017;100(12):2357-2361. 